Nervenarzt 2019 · 90:958-960 https://doi.org/10.1007/s00115-019-0766-7 Online publiziert: 15 . Juli 2019 (c) Springer Medizin Verlag GmbH, ein Teil von Springer Nature 2019
Stefan Unterecker ${ }^{1}$. Gudrun Hefner ${ }^{2}$. Pierre Baumann ${ }^{3}$. Gerd Gründer ${ }^{4}$. Niels Bergemann ${ }^{5} \cdot$ Hans-Willi Clement ${ }^{6}$. Andreas Conca ${ }^{7}$. Jürgen Deckert ${ }^{1}$. Katharina Domschke ${ }^{8,35} \cdot$ Gabriel Eckermann $^{9} \cdot$ Karin Egberts $^{10}$. Manfred Gerlach ${ }^{10}$. Christine Greiner ${ }^{11}$. Ekkehard Haen ${ }^{12}$. Ursula HavemannReinecke $^{13} \cdot$ Renate Helmer ${ }^{14} \cdot$ Ger Janssen ${ }^{15}$. Eveline Jaquenoud ${ }^{16} \cdot$ Gerd Laux $^{17}$. Thomas Messer $^{18} \cdot$ Rainald Mössner ${ }^{19} \cdot$ Matthias J. Müller ${ }^{20} \cdot$ Michael Paulzen $^{21,36}$. Bruno Pfuhlmann ${ }^{22}$. Peter Riederer ${ }^{1}$ - Alois Saria ${ }^{23}$. Bernd Schoppek ${ }^{24}$. Georgios Schoretsanitis ${ }^{25}$. Markus Schwarz ${ }^{26} \cdot$ Margarethe Silva Gracia $^{12}$. Benedikt Stegmann ${ }^{12} \cdot$ Werner Steimer $^{27} \cdot$ Julia C. Stingl ${ }^{11} \cdot$ Manfred Uhr $^{28}$. Sven Ulrich ${ }^{29} \cdot$ Roland Waschgler $^{30}$. Gerald Zernig ${ }^{23,31}$ - Gabriele Zurek ${ }^{32}$. Christoph Hiemke 33,34 $^{3}$

'Klinik für Psychiatrie, Psychosomatik und Psychotherapie, Universitätsklinikum Würzburg, Würzburg, Deutschland; ${ }^{2}$ Waldkrankenhaus Köppern, Vitos Klinik Hochtaunus, Friedrichsdorf, Deutschland; ${ }^{3}$ Klinik für Psychiatrie, Universität Lausanne, Prilly-Lausanne, Schweiz; ${ }^{4}$ Abteilung Molekulares Neuroimaging, Medizinische Fakultät Mannheim, Zentralinstitut für Seelische Gesundheit, Mannheim, Deutschland; ${ }^{5}$ Klinik für Psychosomatik und Psychotherapie, Kitzberg-Kliniken, Bad Mergentheim, Deutschland; ${ }^{6}$ Klinik für Kinder- und Jugendpsychiatrie, Universität Freiburg, Freiburg, Deutschland; ${ }^{7}$ Servizio Psichiatrico del Comprensorio Sanitario di Bolzano, Bolzano, Italien; ${ }^{8} \mathrm{Klinik}$ für Psychiatrie und Psychotherapie, Universitätsklinikum Freiburg, Freiburg, Deutschland; ${ }^{9}$ Psychiatrische Klinik, Kaufbeuren, Deutschland; ${ }^{10} \mathrm{Klinik}$ für Kinder- und Jugendpsychiatrie, Psychosomatik und Psychotherapie, Universitätsklinikum Würzburg, Würzburg, Deutschland; "Bundesinstitut für Arzneimittel und Medizinprodukte (BfArM), Bonn, Deutschland; ${ }^{12}$ Klinische Pharmakologie, Institut AGATE, Pentling, und Klinische Pharmakologie am Lehrstuhl für Pharmakologie und Toxikologie, Universität Regensburg, Regensburg, Deutschland; ${ }^{13} \mathrm{Klinik}$ für Psychiatrie und Psychotherapie, Universität Göttingen, Göttingen, Deutschland; ${ }^{14}$ Zentrum für Epilepsie, Bielefeld, Deutschland; ${ }^{15}$ Medizinisches Labor Stein, Limbach Group, Mönchengladbach, Deutschland; ${ }^{16}$ Psychiatrische Klinik, Königsfelden, Brugg, Schweiz; ${ }^{17}$ Institut für Psychologische Medizin und Zentrum für Neuropsychiatrie, Soyen und Waldkraiburg, Deutschland; ${ }^{18}$ Psychiatrische Klinik, Danuviuskliniken, Pfaffenhofen, Deutschland; ${ }^{19} \mathrm{Klinik}$ für Psychiatrie und Psychotherapie, Universität Tübingen, Tübingen, Deutschland; ${ }^{20}$ Psychiatrische Klinik Oberberggruppe und Justus-Liebig-Universität Gießen, Berlin und Gießen, Deutschland; ${ }^{21}$ Alexianer Krankenhaus, Aachen, Deutschland; ${ }^{22}$ Psychiatrische Klinik Weisser Hirsch, Dresden, Deutschland; ${ }^{23}$ Department für Psychiatrie, Psychotherapie und Psychosomatik, 1, Abteilung für Experimentelle Psychiatrie, Medizinische Universität Innsbruck, Innsbruck, Österreich; ${ }^{24}$ Psychiatrische Klinik, kbo-Isar-Amper Klinikum München-Ost, München-Haar, Deutschland; ${ }^{25}$ Klinik für Psychiatrie und Psychotherapie, Universität Bern, Bern, Schweiz; ${ }^{26}$ Institut für Laboratoriumsmedizin, Klinikum der Universität München, Ludwig Maximilian Universität, München, Deutschland; ${ }^{27}$ Institut für klinische Chemie und Pathobiochemie, Technische Universität München, München, Deutschland; ${ }^{28}$ Max Planck Institut für Psychiatrie, München, Deutschland; ${ }^{29}$ Aristo Pharma $\mathrm{GmbH}$, Berlin, Deutschland; ${ }^{30}$ Psychiatrische Klinik, Feldkirch, Österreich; ${ }^{31}$ Gerichtssachverständiger und Psychotherapeut, Hall in Tirol, Österreich; ${ }^{32}$ Medizinisches Labor Bremen, Bremen, Deutschland; ${ }^{33}$ Klinik für Psychiatrie und Psychotherapie, Universitätsklinikum Mainz, Mainz, Deutschland; ${ }^{34}$ Institut für klinische Chemie und Laboratoriumsmedizin, Universitätsklinikum Mainz, Mainz, Deutschland; ${ }^{35}$ Medizinische Fakultät, Universität Freiburg, Freiburg, Deutschland; ${ }^{36} \mathrm{Klinik}$ für Psychiatrie, Psychotherapie und Psychosomatik, Medizinische Fakultät, RWTH Aachen, Aachen, Deutschland

\section{Erratum zu: Therapeutisches Drug-Monitoring in der Neuropsychopharmakologie}

\section{Zusammenfassung der Konsensusleitlinien 2017 der TDM-Arbeitsgruppe der AGNP}

Die Online-Version des Originalartikels ist unter https://doi.org/10.1007/s00115-018-0643-9zu finden. 


\section{Erratum zu:}

Nervenarzt 2018

https://doi.org/10.1007/s00115-0180643-9

Im Originalbeitrag ist Tab. 2 leider fehlerhaft. Für die Substanzen Carbamazepin und Valproat wurde im Originalbeitrag ein Empfehlungsgrad zur Anwendung des therapeutischen Drug-Monitorings (TDM) von 2 (empfohlen) angegeben. Allerdings besteht für die Durchführung von TDM sowohl für Carbamazepin als auch für Valproat der Empfehlungsgrad 1 (dringend empfohlen). Wir bitten dies zu beachten.

\section{Korrespondenzadresse}

\section{PD Dr. Stefan Unterecker}

Klinik für Psychiatrie, Psychosomatik und Psychotherapie, Universitätsklinikum Würzburg Margarete-Höppel-Platz 1, 97080 Würzburg, Deutschland unterecker_s@ukw.de

\section{Depressionen: Akupunktur überzeugt in Multimodalen Therapien}

Bereits im Jahr 2020 könnten Depressionen weltweit die häufigste bis zweithäufigste Volkskrankheit sein. Mit etwa vier Millionen Erkrankten gehört sie in Deutschland schon heute zu den am weitesten verbreiteten Beschwerden. Nach Meinung und Erfahrung vieler Ärzte und Patienten kann Akupunktur helfen, die Krankheit in den Griff zu bekommen.

Depressionen können sich in unterschiedlichen Formen und Schweregraden zeigen. Lebensumstände, Belastungen, genetische Faktoren, aber auch Jahreszeiten begünstigen - oftmals im Zusammenspieleine Erkrankung. Eine Vielzahl von Depressionssymptomen wird erfolgreich mit Akupunktur behandelt, darunter Nervosität und Schlaflosigkeit, körperliche und geistige Erschöpfung oder übermächtige Traurigkeit.

Als zielführende und heute etablierte Behandlungsmethode gilt die Kombination von Medikation (z. B. durch Verordnung von Antidepressiva) und Psycho- oder Verhaltenstherapien. Dieser sogenannte multimodale Therapieansatz kann darüber hinaus mit heilungsförderlichen Licht-, Ergound Kunsttherapien oder Sport ergänzt werden. Auch die Ohr- und Körperakupunktur zählt zu den verbreiteten Verfahren eines komplementären Behandlungskonzepts. Ihr positiver Einfluss auf die Symptome einer depressiven Erkrankung wurde bereits im Rahmen zahlreicher belastbarer Studien nachgewiesen.

Bei multimodalen Behandlungen, die einen nachweislich langfristigeren Effekt zeigen als Einzeltherapien, überzeugt die fachgerecht durchgeführte Nadelung durch ihre hohe ganzheitliche Wirksamkeit. „Neben einem positiven Langzeiteinfluss profitieren die $\mathrm{Pa}$ tienten vor allem davon, dass die Akupunktur oft eine Reduktion der Medikamentendosis ermöglicht", so PD Dr. med. Dominik Irnich, 1. Vorsitzender der 1951 gegründeten Deutschen Ärztegesellschaft für Akupunktur e.V. ( DÄGfA).

Nicht selten führt die Einnahme von Antidepressiva zu negativen Begleiterscheinungen, darunter Gewichtszunahme, sexuelle Dysfunktionen oder Schlafstörungen. „Diese lassen sich durch Einsatz einer Nadeltherapie deutlich mildern. In Einzelfällen konnte auch beobachtet werden, dass Beschwerden, die als Nebenwirkung mit dem Absetzen von
Medikamenten einhergehen können, wie z.B. der sogenannte Entzugskopfschmerz, gut auf Akupunktur ansprechen.,' erklärt Dr. med. Richard Musil, Facharzt für Psychiatrie und Psychotherapie, Oberarzt der Klinik für Psychiatrie und Psychotherapie an der LMU München.

Das macht die Akupunktur insbesondere dann zu einem wichtigen Baustein in der Behandlung von Depressionen, wenn eine Medikation aufgrund besonderer patientenseitiger Vorbedingungen nur begrenzt durchgeführt werden kann - etwa bei Vorerkrankungen, Unverträglichkeit oder während einer Schwangerschaft.

Studienlage und Praxiserfahrung sprechen dafür, der Akupunktur einen festen Platz im Gesundheitssystem einzuräumen - die Akzeptanz der Nadeltherapie im Bereich der psychiatrischen Therapie ist bei Ärzten und Therapeuten hoch. Gemeinsam mit anderen Fach- und Berufsverbänden setzt sich die DÄGfA deshalb dafür ein, dass die Behandlungsmethode von Politik, Forschung, Verbänden und Ärztekammern stärker und angemessen berücksichtigt wird.

\section{Deutsche Ärztegesellschaft für} Akupunktur (DÄGfA) 
Hier steht eine Anzeige.

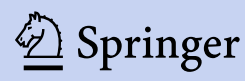

